Euskal ikerketen aldizkaria | Revue d'études basques |

Revista de estudios vascos | Basque studies review

$5 \mid 2000$

Numéro V

\title{
L'autobiographie comme registre littéraire : Ameriketako orhoitzapenak de Jean Etchepare
}

Jean Casenave

(2) OpenEdition

Journals

Édition électronique

URL : http://journals.openedition.org/lapurdum/1320

DOI : 10.4000/lapurdum.1320

ISSN : 1965-0655

Éditeur

IKER

Édition imprimée

Date de publication : 1 octobre 2000

Pagination : 283-292

ISBN : 2-84127-161-7

ISSN : $1273-3830$

Référence électronique

Jean Casenave, «L'autobiographie comme registre littéraire: Ameriketako orhoitzapenak de Jean Etchepare », Lapurdum [En ligne], 5 | 2000, mis en ligne le 01 juin 2009, consulté le 04 juillet 2020. URL : http://journals.openedition.org/lapurdum/1320 ; DOI : https://doi.org/10.4000/lapurdum.1320 


\section{L'autobiographie comme registre littéraire : Ameriketako orhoitzapenak de Jean Etchepare}

Jon Casenave

UMR 5478

Le parti pris autobiographique est quasi inexistant dans la littérature basque classique. Si l'on excepte les allusions et références personnelles disséminées par B. Detchepare, A. Oihenart, Axular ou J. B Elissamburu, les écrivains basques n'ont, jusqu'à la fin du XIX ${ }^{e}$ siècle, guère parlé d'eux-mêmes dans leurs ouvrages. On peut hasarder deux explications à cela : d'abord, il faut se souvenir que la plupart des auteurs classiques et modernes d'expression basque étaient religieux de leur état et donc, de par leur vœu d'humilité, tenus à effacer de leurs écrits une évocation de soi trop personnelle ou susceptible d'apparaître comme complaisante voire impudique. Il faut savoir aussi que l'auteur de langue basque se considérait - et était considéré par le public-comme le porte-voix d'une identité collective et d'une langue menacée. Aussi, face à une situation d'urgence, l'écrivain est amené à revendiquer une reconnaissance de son identité au plan communautaire et non pas personnel.

Dans la culture basque contemporaine, l'expression de soi a largement pris place dans la production littéraire. Elle a même atteint le grand public à travers des livres comme Aihen ahula (1978) de D. Landart et a donné lieu à des expériences littéraires plus confidentielles mais très originales à l'image du Denbora galdu alde (1987) de B. Gandiaga.

Si aujourd'hui le registre autobiographique semble largement admis dans le cadre général de l'expression littéraire en langue basque, il est intéressant d'observer comment et à quel prix s'est opérée cette transition. À ce sujet, l'exemple le plus significatif nous est, sans nul doute, fourni par Jean Etchepare au tout début du XX $X^{\circ}$ siècle. L'utilisation qu'en fait Jean Etchepare dans Buruchkak, son premier livre publié en 1910, ne manquait ni d'originalité ni d'audace. À une époque où il est du devoir de l'écrivain de s'effacer devant une identité collective, Jean Etchepare place pour une grande part, son premier ouvrage dans le registre autobiographique. La démarche de l'auteur de Buruchkak est donc à la fois surprenante et novatrice dans le cadre de la littérature basque du début du siècle puisqu'il mêle dans son livre l'expression courante d'une identité collective et d'une différenciation culturelle à une forte revendication individuelle. 
À la lecture de ce livre on peut penser que le parti pris autobiographique relève de l'artifice formel, un artifice propre à fournir une organisation d'ensemble et un fil conducteur à une série de textes fort divers tant par les thèmes abordés que par les genres et les tons utilisés.

Au-delà de cette facilité de forme, il faut s'interroger sur une motivation beaucoup plus profonde, intimement liée à l'ambition perceptible chez Jean Etchepare de situer son ouvrage dans le domaine littéraire et plus seulement journalistique, ceci dès lors qu'il décide de passer de la chronique hebdomadaire à la publication de son premier livre. Par son ouvrage, il porte une bonne partie de son histoire personnelle à la connaissance du public, manifeste le désir de se faire connaître et reconnaître à travers elle. Ce recours délibéré à des éléments privés lui permet tout à la fois d'afficher sa vocation d'écrivain et d'ouvrir une nouvelle voie à la création littéraire en langue basque, à savoir celle de l'autobiographie. Ainsi, le choix du registre autobiographique peut être interprété comme un indice de littérarité, une marque de reconnaissance du fait littéraire que le jeune écrivain affiche dès l'ouverture de son livre.

\section{La dimension autobiographique de Buruchkak}

Il faut tout d'abord rappeler que Buruchkak (1910) est un recueil d'essais qui rassemble vingt-six textes relativement courts, parus pour la plupart sous forme d'articles ou de feuilletons dans les hebdomadaires Eskualduna et Eskualdun ona entre 1904 et 1909. Consacrés aux questions culturelles, éducatives ou même économiques relatives au Pays basque, ces articles prennent la dimension d'essais littéraires grâce à divers procédés de distanciation par rapport à l'actualité immédiate. Un fait n'a pu manquer d'étonner le lecteur bascophone de 1910, familier des publications de son époque : neuf des vingt-six textes qui composent Buruchkak sont donnés comme autobiographiques.

Il faut cependant ajouter que l'utilisation du genre autobiographique que fait Jean Etchepare ne correspond pas strictement aux définitions fermées que donnent Jean Starobinski' ou Philippe Lejeune ${ }^{2}$. Le premier indique qu'il s'agit « de la biographie d'une personne faite par elle-même ». Philippe Lejeune, quant à lui, s'est efforcé d'analyser les caractéristiques du genre à travers l'étude de quelques textes fondateurs. Il en a tiré la définition suivante :

Récit rétrospectif en prose qu'une personne réelle fait de sa propre existence lorsqu'elle met l'accent sur sa vie

individuelle, en particulier sur l'histoire de sa personnalité.

Du point de vue générique, les textes que J. Etchepare rassemble dans son premier livre se rapprochent davantage des genres « essais et souvenirs ». Néanmoins, conformément aux règles de l'autobiographie, il y a bien identité de l'auteur et du narrateur. En revanche, le narrateur n'est pas forcément le personnage principal du récit. En fait, il est plus souvent en position d'observateur et de rapporteur de la scène, de l'incident ou du débat que d'acteur essentiel au déroulement de l'intrigue. 
Ainsi, il propose des textes qui sont rédigés au passé et évoquent des événements personnels (ex : « Ameriketako orhoitzapenak » ou « Biritchiak ») ou collectifs (ex : " Pilota partida » ou « Ongi etorria »), tous bien fixés sur l'échelle du temps. Ce passé peut donc être d'ordre individuel, familial ou collectif, mais dans tous les cas, son évocation induit un traitement littéraire. En effet, l'option choisie n'est jamais celle d'une reconstitution historique objective mais bien celle du récit ou du compte rendu subjectif. Ce point est souligné par la présence dans tous ces textes écrits au passé des marques de la première personne (du singulier ou du pluriel). Ce choix énonciatif s'accompagne de nombreux procédés de modalisation et l'introduction du vocabulaire affectif ajoute à la subjectivité d'un propos marqué, le plus souvent, par une inflexion lyrique.

Enfin, le recours au passé entraîne nécessairement un effort de recomposition de la réalité qui, renforcé par l'intrusion de la subjectivité, indique un registre littéraire. Le narrateur signale ce processus de reconstruction a posteriori dans «Ameriketako orhoitzapenak » :

\section{Orhoitzapen chumeak, bizkitartean, ta nork daki? geroztikako zenbaitekin nahastekatuak orobat, nehork ez baitu ikusten nola dabilkion bere barnea... Halere begiratu ditudan bezala ezarriko ditut hemenche.}

"Ameriketako orhoitzapenak », p. 8.

Des souvenirs tout simples cependant, et qui sait? en même temps mêlés à d'autres plus récents, car personne ne voit comment fonctionne sa conscience... Toutefois, je les transcrirai ici comme je les ai retenus.

Ce parti pris de sincérité et de vérité correspond à un pacte ou à un contrat de lecture que l'écrivain souhaite passer avec son interlocuteur. Bien que l'auteur semble s'en défendre, cette remarque indique aussi qu'il y a eu recomposition du passé, donc, nécessairement, interprétation. La même affirmation de sincérité se retrouve dans «Pilota partida ». Jean Etchepare signale en note qu'il s'est efforcé de rendre au mieux ce qu'était, dans sa jeunesse, une partie de pelote.

Parmi les neuf textes évoqués plus haut, quatre essais font explicitement référence à l'enfance d'un narrateur qui possède toutes les caractéristiques biographiques de l'auteur. En ouverture du livre, l'écrivain nous raconte les souvenirs de sa petite enfance passée dans la pampa argentine, au milieu des moutons et des chevaux élevés par son père ("Ameriketako oroitzapenak »). Les trois autres textes ont Mendionde pour cadre et sont donc postérieurs au retour en Europe de la famille. Dans ces trois brefs récits, l'écrivain met en scène respectivement une partie de pelote très disputée qui resta longtemps dans les mémoires (« Pilota partida »), la mort d'un chien enragé tué par son propre maître, le fabricant de clous voisin de la famille Etchepare ("Itze-egilearen zakurra »), et enfin, la réception solennelle réservée par le village de Mendionde à son nouveau curé ( «Ongi etorria »). Un quatrième récit situé également à Mendionde, «Biritchiak», est lui postérieur à cette période puisque le narrateur est déjà un jeune homme engagé dans des études supérieures à Bordeaux. 
Il faut maintenant se demander quel est, sur le plan littéraire l'intérêt de cette ouverture à l'autobiographie. En effet, à y regarder de près, on voit bien que Jean Etchepare livre peu de détails sur lui-même et les éléments d'histoire personnelle qu'il donne de façon ouverte sont limitées à l'enfance et à l'adolescence. Dans ces conditions, pourquoi parler de soi ?

\section{"Ameriketako orhoitzapenak »": le récit d'enfance}

L'essai qui ouvre le recueil, " Ameriketako orhoitzapenak », est à ce titre tout à fait significatif, d'autant qu'il est, avec « Itze-egilearen zakurra », l'un des deux extraits relevant stricto sensu du genre autobiographique. Selon ses propres termes, Jean Etchepare y rassemble tous ses souvenirs d'Amérique. À partir de ce fragile matériau, il reconstitue quelques bribes de sa petite enfance - l'auteur a six ans lorsqu'il quitte l'Argentine pour s'installer définitivement en Europe avec sa famille -, la vie de ses proches et celle de l'exploitation familiale en Argentine.

Ce récit d'enfance esti, sans conteste, l'un des textes-clés du recueil. Bien sûr, cela tient au fait qu'il se trouve placé en ouverture du livre et qu'il impose d'emblée la présence d'un narrateur-commentateur. Il est également patent que ce recours initial au registre autobiographique place immédiatement l'ouvrage en décalage par rapport aux créations antérieures comme contemporaines dans lesquelles l'expression du moi est quasiment inexistante.

Mais l'intérêt de ce texte réside aussi dans le fait qu'il se présente à la fois comme le plus personnel et le plus littéraire du livre. Ces lignes que Jean Etchepare consacre à ses premières années constituent l'un des premiers et des rares témoignages de l'existence des Basques en Amérique. Au moment où l'écrivain l'aborde, ce sujet est capital pour l'avenir de la littérature basque même si l'on peut constater aujourd'hui, avec un siècle de recul, qu'il s'agit bel et bien d'un rendez-vous manqué. Jusqu'à cette date, il a donné essentiellement lieu à un traitement négatif dans le discours identitaire et ce, de la part d'auteurs restés en Europe qui souhaitaient dissuader les jeunes gens de prendre le bateau pour La Plata ou les autres contrées du Nouveau Monde. À leur décharge, il faut préciser que le programme des Jeux floraux institués par Antoine d'Abbadie en 1853, principal soutien de la production poétique de ces années-là, encourageait cette vision pessimiste ${ }^{3}$ en demandant «... une chanson exprimant les regrets d'un Basque en partance pour Montevideo ».

Pourtant, compte tenu de l'impact humain et économique du phénomène ainsi que des potentialités poétiques et esthétiques contenues dans cette ouverture à de nouveaux horizons, la littérature basque pouvait trouver un souffle nouveau dans cette expérience de l'émigration. En fait, c'est d'Amérique que vient le renouveau attendu avec la publication par Jose Mendiague en 1900 à Buenos Aires d'un recueil de chants intitulé Zazpiak bat. Eskualdun Kantuak ${ }^{\dagger}$. Dans la génération suivante ce sont Jean Etchepare et Pierre Lhande, des enfants « d'Américains » qui abordent la question du Nouveau Monde sous un jour plus favorable. Le premier nommé livre en 1910 une étude savante (L'Emigration basque) qui fait la part des 
choses et replace le phénomène dans une perspective historique et psychologique, parfois hasardeuse du reste pour ce qui est du dernier aspect. Quant à l'auteur de Buruchkak, il en donne une version narrative ${ }^{5}$ en évoquant l'épisode argentin de la vie de sa propre famille.

Cependant, contre toute attente à une époque où la littérature basque était peu portée à la personnalisation de l'écrit, il ne propose pas un récit neutre et réaliste de l'existence des colons basques même si son texte fourmille de détails précis. Son entreprise prend une orientation nettement littéraire grâce au point de vue narratif qu'il a choisi et à la mise en valeur d'une vision intimiste de la réalité, néanmoins toujours proche du quotidien. Il prend le parti de raconter la vie des émigrants à travers le regard du petit enfant qu'il était à ce moment-là de sa propre existence.

En recomposant ce passé lointain à l'âge de trente ans, il aurait pu mettre à profit les souvenirs de ses parents, oncles et tantes pour écrire une évocation fidèle de cette époque révolue à la troisième personne du singulier, sous la forme d'un essai ou d'un récit. Mais son projet ne se limite pas à cette reconstitution de l'histoire familiale ou plutôt, celle-ci constitue le prétexte et le sujet d'une entreprise autobiographique qui lui permet d'ouvrir un nouvel espace littéraire. En choisissant de rédiger les souvenirs de sa première enfance, il change de registre et détourne partiellement le sujet de l'Amérique au profit d'une recherche davantage tournée vers son expérience personnelle que vers la dimension géographico-historique de la situation décrite. Il propose une vision originale du monde et, fait intéressant pour la littérature basque, il crée le modèle de langue et l'imaginaire qui sont nécessaires à son expression.

L'insistance sur la vie intérieure du petit enfant est le meilleur symbole de ce choix narratif particulier car, par cet artifice, il propose un angle d'observation inédit. Il s'attache à dépeindre les personnages d'en dessous, en référence à sa petite taille et à ses préoccupations d'enfant :

... arratsalde aphala zen; ama eiche sahetsean jarria, itzalean ; ni haren inguruan josteta. Horra nun ikusten dugun aita gure ganat heldu, begitarte mokorrarekin arabez, ezen berehala amaren altzorat ihes egin nuen.

«Ameriketakoorhoitzapenak », p. 17.

... c'était la fin de l'après-midi ; maman était assise sur le côté de la maison, à l'ombre ; moi, je jouais autour d'elle. Soudain nous voyons papa venir vers nous, le visage en apparence fầché, sí bien que je me réfugiai aussitôt sur les genoux de ma mère.

Comme le fait à la même époque Marcel Proust dans ses premiers écrits autobiographiques (Jean Santeuil), puis plus tard dans le cycle de « la Recherche", Jean Etchepare s'efforce de retrouver l'impact de ses émotions d'enfant, sa capacité d'émerveillement comme d'effarement, ses joies aussi bien que ses terreurs. Ce travail sur la mémoire le conduit à présenter un tableau réellement atypique dans la production littéraire basque de l'époque 6 . Il rend compte subtilement de ce que l'on pourrait appeler aujourd'hui une problématique œdipienne à travers l'évocation de ses parents. 
Pour l'enfant, la figure maternelle est, conformément à la vérité biographique, très jeune, belle et proche tant par l'âge que par les sentiments. En effet, dans " Ameriketako orhoitzapenak », elle est cette présence à la fois douce et solaire que l'auteur décrit au travail, nimbée de la iumière matinale :

Ene ama maite, ene haurtasuneko argi eta gozo bakarra, bethi ikusten dut, etche sahetsean chutik, goizarekin, chuhailez jauntzia, besoak ukondoraino agerian, eskuen artean oihal zerbait marruskatzen ari [...]

Elhe emaiten zaundan amak, irriño batek arraitzen zakolarik bere begitarte gazte pollita, ahoko sendoak lerro lerro agerian. Nechkato larri bat zitzautan, bainan ona ene alderat ezin gehiago eta, bethi maldatzen ninduelakotz,

halako herabe batekin, nitaz baino gorago naukana.

"Ameriketako orhoitzapenak », p. 12.

Ma chère mère, la seule lumière et la seule consolation de mon enfance, je la vois encore, debout sur le côté de la maison, dans la matinée, vêtue de clair ; les bras nus jusqu'au coude, en train de tordre quelque pièce de linge entre les mains [...]

Ma mère me parlait, tandis qu'un sourire éclairait son jeune et joli visage, montrant ses dents saines et alignées. Elle m'apparaissait comme une grande jeune fille, mais excessivement bonne à mon égard et, parce qu'elle me protégeait toujours, que je tenais pour supérieure à moi, avec une forme de timidité.

Il est frappant de constater que, contrairement à ce qu'on peut lire chez les autres auteurs de la même époque, l'image maternelle s'impose d'abord par sa présence physique. Les qualités de cœur, bien qu'essentielles, n'interviennent qu'après dans la description. L'écrivain s'attache à rendre ce personnage proche, tel qu'il le ressentait lui-même. Pour ce faire, il s'efforce de retrouver des détails très précis, ceux-là mêmes qui l' ont fasciné enfant, comme l'éclair des dents blanches et saines entrevu dans le sourire ou sa gestuelle de lavandière. Il s'efforce également de retrouver et de transmettre ces émotions et ces sentiments forts qui unissaient la jeune mère et son fils aîné. Avec une grande économie de mots, il exprime notamment cette réserve, cette complicité muette et pudique qui caractérisaient leurs relations à cette époque.

Cette mère aimante, seule à lui parler dans un univers familial bouleversé par l'exil', le choie et le protège de la menace paternelle toujours présente. Impitoyable avec le personnel (il a renvoyé son propre beau-frère) comme avec les animaux (il tue les chiens qui s'attaquent aux troupeaux), le père est cette autorité toute puissante, à la fois admirée et redoutée.

C'est bien le regard d'un garçon de six ans qui prévaut dans cette description du père qui devient difficile à comprendre si l'on a oublié cette question du point de vue. A quelques lignes d'intervalle, on trouve, en effet, l'illustration des deux regards portés sur ce personnage. Le premier est celui de l'enfant qui regarde, impuissant, ce père brutal et insensible à sa détresse tuer son chien préféré, le compagnon de ses promenades de petit garçon solitaire :

Kurubilkatu nintzan amari kontra, hatsa doidoi hartzen nuela hersturarekin. Banakien etzuela aitak barkatuko; haren begietan ez nintzela deusik; lehenago egitate berarentzat bazituela zakur andana bat hilik. [...] 
Chimichta bezala iragan ziren denak : aitaren beso agertzea, tiroa, zakurraren erortzea sahetsaren gainerat, sabela zilaturik.

Bizia ordu berean nihauk galtzen nuela iduritu zitzautan...

"Ameriketako orhoitzapenak », p. 18-19.

Je me blottis contre maman, et ne respirai qu'à peine, saisi d'angoisse. Je savais que papa ne pardonnerait pas, que je n'étais rien à ses yeux, et qu'il avait déjà tué de nombreux chiens pour le même fait. [...]

Tout se déroula en un éclair : l'apparition du bras de mon père, le coup de feu, le chien qui tombe sur le côté, le ventre transpercé.

Il me sembla que je perdais moi-même la vie à cet instant.

Plus loin, c'est le narrateur devenu adulte qui, encore à propos d'un chien, évoque d'autres rapports avec son père :

Bazen bertze zakur bat, gogoan bizirik begiratu dutana. Bainan, ez denez bitchikeria! ez nintzan haren izenaz orhoituko, aitak ez balaut geroztik aiphatu, erranez ez duela zakur hura bezain maiterik ukan bihirik, Ameriketan egon den ephe guzian.

"Ameriketako orhoitzapenak »,p. 19.

Il y avait un autre chien dont $j$ 'avais conservé le souvenir. Mais, n'est-ce pas une chose étrange ! je ne me serais pas souvenu de son nom si papa ne m'avait pas parlé de lui bien plus tard, en disant qu'il n'a aimé aucun chien autant que celui-là, durant tout son séjour en Amérique.

Cet incontestable adoucissement de l'image paternelle reste cependant relatif car l'auteur précise :

Hain barna sarthua ukan dut zainetan, ttipi danik, aitaren alderateko beldur hori non, gizondu ta, jarraiki baitzaut, halako uzkur eta, zer nahitarat abia nindadien, huts egiteko ikhara bat jauzarazten zaundala...

"Ameriketako orhoitzapenak », p. 10.

Cette peur à l'égard de mon père a si profondément pénétré dans mes veines que, passé à l'âge adulte, elle m'a accompagné, provoquant en moi une sorte de retenue craintive et quoi que j'entreprisse, la hantise de mal faire.

On se souvient que la « légende familiale » attribue à l'insistance de ce père autoritaire la décision du retrait de Buruchkak prise par l'écrivain, l'année même de la sortie du livre.

Cette pratique du double point de vue et du décalage temporel dans le récit, ce sens de la mesure dans le traitement des personnages et de la réalité et, enfin, la recherche d'un regard intériorisé fondent la singularité de cet essai. Dominée par la présence des deux grandes figures parentales, cette période argentine condense donc quelques-unes des expériences affectives que l'autobiographe pressent comme fondatrices de sa personnalité.

Sans insister outre mesure sur le sujet, on peut mentionner également l'apprentissage de la dépossession et de la souffrance vécu à l'occasion de la perte de son chien préféré « Garibaldi », tué sous ses propres yeux par son père ${ }^{8}$. L'enfance argentine, c'est encore ce temps de liberté aux limites imprécises qu'il occupe à parcourir la plaine à la recherche du tatou, l'animal mythique dont il connaît tous 
les secrets mais qu'il ne parvient pas à capturer. Pour rendre perceptible cette atmosphère si particulière, l'écrivain travaille à une poétisation de l'espace. Cette dernière est particulièrement sensible à travers l'effacement des contours du monde réel. On ne semble pas pouvoir trouver de bornes à la pampa, cette plaine ouverte :

Begiz ikus ahal urrun hedatzen zen zelhaia, belharrez musker gizen, han hemenka bizkitartean pentoka larrutuño batzuez titatua. Zeru aphal urdin batek leherturik bezala zaukan; odaiertzean, urrutirat, ukurtuz haren ganat jausten zela iduri baitzuen. Nun nahi burua goiti zindezan, erdiz erdi aurkitzen zinuen iguzkia, zabal, churi, dichtirantarekin lilugarri, chipilzeko sapa zariola. "Ameriketako orhoitzapenak", p. 10.

La plaine s'étendait à perte de vue, couverte d'une herbe verte et grasse, tachetée pourtant çà et là de petites buttes pelées. Un ciel bas et gris la tenait comme écrasée ; à l'horizon, au loin, on aurait dit qu'il descendait vers elle en se penchant. Où que vous leviez la tête, vous vous trouviez face au soleil, immense, blanc, enchanteur avec ses reflets, qui dégageait une chaleur étouffante propre à vous griller.

Dans ce paysage démesuré, les indications temporelles ordinaires trouvent une élasticité hors du commun. Ainsi, l'enfant passe ses journées dans la plaine, sans autres repères que le soleil et la sensation de faim qui tenaille son estomac. L'Amérique et la vie de la diaspora sont des espaces vierges pour la littérature basque. Sans tomber dans un exotisme maniéré, Jean Etchepare rend perceptible l'étrangeté radicale et l'immensité de ce nouveau monde si différent du Pays basque. En mêlant le regard de l'enfant aux caractéristiques du paysage argentin, il donne l'impression que le temps et l'espace s'allongent infiniment jusqu'à constituer un réel fantasmatique.

Est-ce à dire que ce récit d'une enfance américaine se résume à une évocation du paradis perdu ? De prime abord, on pourrait le penser car le texte est dès les premières lignes caractérisé par une tonalité lyrico-élégiaque :

Gogoa bethea daukat orduko zerez. O haurreko egun berriz jinen etzaretenak, zuetarik dut bihotzean nere bizi apurrak eman daukedan gozorik hoberenak. "Ameriketako orhoitzapenak », p. 7.

J'ai l'esprit rempli des choses de ce temps-là. Oh ! jours d'enfance qui ne reviendrez plus, c'est de vous que je tiens le plus agréable de ce que ma courte existence a pu me donner !

(Traduction de J.B. Orpustan, extraite de Basque et français, p. 193.)

Le recours à des formes rhétoriques qui mettent en valeur l'exclamation, l'apostrophe et même l'incantation donne une orientation nostalgique à l'ensemble. Mais l'expression de la douleur et de l'angoisse vient brouiller ce modèle élégiaque et nuancer cette impression de bonheur idéalisé.

Enfin, Jean Etchepare introduit dans son texte un autre élément de décalage qui trouble le modèle du récit de vie classique. En l'occurrence, il s'agit d'une interrogation sur les tours et les détours de la mémoire. Il parsème sa narration de petites remarques qui créent, en plus des quelques trente années de décalage, une distance entre les faits et l'auteur : 
Ez da deus garbiagorik ene buruan. p. 7.

Il n'y a rien de plus clair dans ma tête...

Han ikusten ditut oraino ardiak... p. 8.

Je vois encore les brebis là-bas...

Begien aintzinean daukat... p. 9.

J'ai devant les yeux...

À côté de ces notations données pour certaines, on trouve des hésitations, des doutes et des questions :

Haur zotz izana gatik, joiten othe ninduenez... baditeke, bainan ez dut gogoan. p. 10 .

Me battait-il quoique je fusse encore un tout petit enfant... c'est possible, mais je ne m'en souviens pas.

Nola othe ditake beraz itchura hori ene orhoitean? Nork erran dezake nola dabilan gure buru barneko alha? p. 21.

Comment cette image se trouve-t-elle donc, dans ma mémoire?

Qui peut dire comment fonctionne notre conscience ?

À travers ces indications, Jean Etchepare laisse entendre qu'il a effectué tout un travail sur ses souvenirs, une reconstruction et une recomposition d'ensemble issues de l'écriture. En effet, cette variété des points de vue, cette mise en valeur esthétique de l'espace et du temps mais aussi des gestes et des activités de la vie quotidienne débouchent autant sur une recréation poétique de l'univers de l'enfance que sur l'évocation de la vie réelle des émigrants basques en Argentine.

Ce récit est placé en tête de l'ouvrage. D'emblée, l'auteur de Buruchkak propose un texte difficile qui ne dit pas exactement ce qu'il est. Il entretient une certaine ambiguité par rapport au réel, ne révèle pas ses intentions et prive le public d'un parcours de lecture clairement balisé. On se trouve bien dès les premières pages du recueil dans une configuration littéraire mise en lumière par Philippe Lejeune dans ses ouvrages :

...le paradoxe de l'autobiographie littéraire, son essentiel

double jeu, est de prétendre être à la fois un discours

véridique et une cuvre d'art.

Moi aussi, p. 26.

L'introduction du discours autobiographique dans Buruchkak témoigne donc autant du désir de changer la manière d'aborder le texte littéraire que de la volonté de se raconter soi-même. Le discours autobiographique est l'occasion pour Jean Etchepare de mettre en scène des émotions et des sentiments que les auteurs s'appliquent à garder dans l'ombre au nom d'un cliché qui prête aux Basques une extrême pudeur. Dans Ramuntcho, Pierre Loti a repris cette idée aux commentateurs antérieurs et a contribué de façon décisive à accroître sa popularité :

Pour accueillir et embrasser son fils, elle sourit de joie et de tendresse; mais, silencieux par nature, renfermés tous deux, ils ne se disaient guère que ce qu'il était utile de se dire. Chapitre 1.

Itchoua, lui, le chef, écoute plus qu'il ne parle... Chapitre 4. 
En effet, on retrouve cette même notion chez des romanciers comme Domingo Agirre (Garoa) ou Jean Barbier (Piarres) lorsqu'ils évoquent les relations à l'intérieur de la famille rurale. Comme le souligne aussi Pierre Lhande dans Autour d'un foyer basque, cette retenue concerne les gestes ( On a reproché aux Basques de ne jamais donner le bras à leurs femmes... », p. 78) et les paroles («... la maîtresse de maison [...] accable des preuves - mieux que tangibles - de son activité féconde, les passifs convives qui la louent d'un murmure grave et satisfait », p. 77), tout le domaine des sentiments est décrit comme empreint de réserve. Pour sa part, Jean Etchepare a déjoué le stéréotype en recourant à un souvenir personnel dont le caractère anecdotique permet de suggérer l'émotion sans la souligner à gros traits.

\section{Notes}

1. Jean Starobinski, dans le $n^{\circ} 3$ de la revue Poétique, Seuil, 1970.

2. Philippe Lejeune, Le pacie autobiographique, Seuil, 1975.

3. Dans L'auvre poétique de Etchahun (Bilbao I970, p. 448 à 471), Jean Haritschelhar propose un dossier complet sur cette question (libellé du sujet, annexes et commentaires).

4. Pierre Charritton a préparé et présenté la nouvelle édition de ce volume : Jose Mendiague (18451937) Donostia, Etor, 1992.

5. Jean Etchepare a semble-t-il toujours considéré qu'il y avait dans l'imaginaire américain des Basques matière à écrire une grande œuvre. Après la mort du romancier Domingo Agirre, il réaffirme, avec déjà une pointe de regret, son vau de lire un jour l'ouvrage que lui-même n'a pas pu écrire sur le sujet :

Bizia luzatu balitzakio (...), baditeke xehatuko zauzkigun, beti

kondairaz apainduz, Eskualdunen lanak itsasoaz harainkilo

leihorretan : eskualdun idazle batek asma dezaken lanik ederrena osatu

baitzuken orduan Agirrek 1932, Gure Herria, repris dans le tome V des œuvres complètes, p. 140.

Si sa vie s'était s'était prolongée, il se peut qu'il nous aurait décrit, toujours dans le style romanesque, les travaux des Basques dans les territoires d'outre-mer : Agirre aurait sans doute alors accompli la plus belle cuvre que puisse imaginer un écrivain basque.

6. On peut considérer qu'il faut attendre l'époque contemporaine pour trouver une vision aussi subtile des conflits intérieurs et, notamment, le témoignage autobiographique de Bitoriano Gandiaga, Denbora galdu alde, Donostia, Erein, 1985.

7. Dans la maison-souche, plusieurs générations se côtoient et les enfants sont volontiers confiés aux grands-mères ou aux tantes, d'ordinaire plus disponibles que les mères occupées aux travaux extérieurs.

8. Ce motif revient également ) la fin de « itze-egilearen zakurra ». Dans cet autre récit autobiographique, le chien meurt des mains du cloutier et non de celles du père de l'auteur mais les deux figures ont beaucoup de points communs. Sans parler de motif obsessionnel, on peut toutefois noter l'aspect symbolique de cette reprise. 